\title{
Abstracts from the ASENT 14th Annual Meeting
}

\section{Genetic Ablation or Pharmacological Inhibition of Cell Cycle Pathways Provides Neuroprotection in Experimental TBI}

Shruti V. Kabadi, PhD, Bogdan A. Stoica, MD, Shihong Li, MD PhD, Marie Hanscom, BS, Alan I Faden, MD, Center for Shock, Trauma and Anesthesiology Research (STAR), National Study Center for Trauma and EMS, University of Maryland, School of Medicine; Kimberly R. Byrnes, PhD, Department of Anatomy, Physiology and Genetics, Uniformed Services University

Introduction: Traumatic brain injury (TBI) induces biochemical and molecular changes that contribute to delayed, secondary tissue damage. We have proposed that TBI induces cell cycle activation (CCA) that leads to secondary damage by causing caspase-dependent apoptosis of postmitotic cells (neurons, oligodendroglia), and proliferation/ activation of mitotic cells (astroglia, microglia). Recently we demonstrated that post-injury treatment with cyclin dependent kinase inhibitors (CDKI) attenuates CCA and improves functional recovery and histological outcomes after fluid percussion induced TBI in rats.

Objective: In the present study, we compared the neuroprotective effects of cell cycle inhibition in a wellcharacterized mouse controlled cortical impact (CCI) model using either genetic ablation of the critical upstream cyclin D1 (CD1 knockout, $-/-$ ) or central administration of the selective CDKI, Roscovitine.

Methods: Behavioral assessments of motor (beam walk) and cognitive (Morris water maze and novel object recognition) functions were performed after CCI; animals were euthanized at 24 hours, 7 or 21 days after injury for histological evaluation (lesion volume, stereological assessments of neuronal cell loss in hippocampus, and microglial activation in cortex).
Results: CD1 -/- or treatment with Roscovitine significantly attenuated motor deficits, improved cognitive (spatial working and retention) recovery and decreased lesion volume. In addition, both strategies of cell cycle inhibition limited cell loss in dentate gyrus (DG), which showed a strong correlation with the improved cognitive function, and significantly attenuated post-traumatic microglial activation.

Conclusion: Our results, using complementary genetic and pharmacological strategies support a role for CCA in the pathophysiology of secondary injury after CCI. Therefore, interventions targeting CD1 or inhibiting CDKs may provide novel and effective therapeutic approaches for the treatment of TBI.

Grant Support: This study was supported by National Institutes of Health Grant no. NS052568 to Dr. Faden Competing Financial Interests: The authors declare no competing financial interests.

Delta Opioid Receptor \& 5HT3 Receptor Internaction: A Novel Mechanism

Kartic Rajput, MD, PhD, Department of Psychiatry \& Behavioral Sciences, Tulane University; Lerna Minor, Dennis Paul, Department of Pharmacology, LSU Health Sciences Center

Introduction: About one in four people suffer from moderate to severe pain. Still the under-treatment of pain remains a major issue due to abuse liability of opioid. The separation of addiction issues from pain management could be the answer for adequate pain control. This can be achieved by understanding and employing signal integration in drug-drug interactions. The mechanism and site of interaction between opioid and serotonergic systems are poorly understood. Both opioid and 5-HT3 receptors are found on the terminals of the sensory primary afferent 
neurons in the dorsal horn of the spinal cord. Also, the stimulation of both DOP and 5-HT3 receptor produces analgesia but not reinforcement.

Methods: We examined the interaction using '-opioid and 5-HT3 receptor agonist in NG108-15 cell line and CD-1 mice. DOP receptor affinity and function was studied in NG108-15 cell line using a radioligand binding assay and cAMP assay respectively. Physiological interaction was studied in male CD-1 mice. DOR \& 5HT3 agonists were given by intrathecal injections and nociception was evaluated by the tail-flick assay in male CD-1 mice. Results from the tail-flick assay were analyzed by isobolographic analysis as described by Tallarida et al.

Results: In binding assays, the affinity of DOR to its agonist was enhanced four fold in the presence of 2-methyl5HT, a 5HT3 agonist. Also, 5HT3 receptor stimulation potentiated the inhibitory effect of DOR, a G-protein coupled receptor, on forskolin stimulated cAMP production. In CD-1 mice, intrathecal administration of both DOP receptor agonists and 5HT3 receptor agonists produced dose-dependent analgesia. The co-administration of DOP and 5HT3 receptor agonists produce analgesic synergy about 10 -fold greater than additive.

Discussion: The interactions between the DOP and 5HT3 receptors is not limited to changes in binding affinity and cell signaling but can also produce a synergistic interaction at a physiological level. The synergistic combinations provide the opportunity to reduce the dosage of both drugs therefore, decreasing adverse effects including reinforcing properties. In conclusion, this information will facilitate the development of logical drug combinations with better efficacy or fewer adverse effects not only in the field of pain management but also in other medical specialties.

\section{Combined Inhibition of Caspase-dependent and Caspase-independent Cell Death Pathways Affords Additive Neuroprotection after Experimental Traumatic Brain Injury}

Chun-Shu Piao ${ }^{1}$, MD, PhD, David J. Loane ${ }^{1}, \mathrm{PhD}$, Bogdan A. Stoica ${ }^{1}, \mathrm{MD}$, Shihong $\mathrm{Li}^{1}, \mathrm{MD}, \mathrm{PhD}$, Marie Hansco ${ }^{1}$, BS, BA, Klas Blomgren ${ }^{2}$, MD, Alan I. Faden ${ }^{1}$, MD.

${ }^{1}$ Center for Shock, Trauma and Anesthesiology Research (STAR) and Department of Anesthesiology, University of Maryland, School of Medicine;

${ }^{2}$ Centre for Brain Repair and Rehabilitation, Institute of Neuroscience and Physiology, Goteborg University, Goteborg, Sweden.

Background: Delayed neuronal cell death is a key component of secondary tissue damage after traumatic brain injury (TBI) and involves multiple independent pathways- two of the most significant being caspasemediated and apoptosis inducing factor (AIF)-mediated (caspase-independent) mechanisms. We examined whether combined inhibition of these pathways provides additive neuroprotection. The peptidyl-proplyl isomerase, Cyclophilin A (CypA) transports AIF from the cytosol to the nucleus and is required for AIF-dependent cell death. We investigated whether CypA gene knockout (KO) together with a pan-caspase inhibitor, boc-aspartyl(OMe)-fluoromethylketone (BAF), show improved outcomes after TBI as compared to $\mathrm{KO}$ or BAF treatment alone.

Methods: Adult WT or CypA KO mice were subjected to moderate controlled cortical impact (CCI) followed by administration of either BAF (300 nmols) or equal volume vehicle by icv injection 30 min after injury (each $n=12$ per group). Motor function using a beam walk task and spatial learning/memory performance using the Morris water maze were assessed. Lesion volume was examined by T2weighted MRI at $21 \mathrm{~d}$. Fodrin cleavage fragments western blot, AIF/CypA and AIF/H2AX co-immunoprecipitation, and immunofluorescent staining were employed for evaluation of mechanisms involved.

Results: Both vehicle-treated CypA KO mice and BAFtreated WT mice showed improved motor function and cognitive performance compared to vehicle-treated WT controls. CypA KO mice that received BAF showed improved motor and cognitive outcomes, as well as reduced lesion volumes, compared to $\mathrm{KO}$ or BAF treatment alone. The caspase-specific fodrin cleavage fragments were attenuated by BAF treatment and the $\mathrm{AIF} / \mathrm{H} 2 \mathrm{AX}$ interaction was reduced in the CypA KO animals, illustrating the specificity of the interventions. AIF nuclear translocation was decreased in CypA KO mice, whereas TUNELpositive apoptotic bodies were reduced by BAF-treatment. Conclusion: These data indicate that both AIF-dependent and caspase-dependent pathways play important, independent roles in secondary injury after CCI, and suggest that combined therapeutic strategies directed at multiple programmed cell death pathways may provide superior neuroprotection than those directed at single mechanisms.

Acknowledgments: This work was supported by NIH grant 5R01NS037313-08.

\section{SyNAPSe ${ }^{\circledR}:$ Study BHR-100-301, a Phase 3 Study of Progesterone in Severe Traumatic Brain Injury (TBI)}

Neta Reich Nelson, MPH, Vadim Paluy, MD, Thomas MacAllister, PhD, BHR Pharma, LLC

Introduction: Numerous preclinical studies have demonstrated that progesterone has beneficial effects in TBI through multiple mechanisms. In a pilot and two Phase 2 
studies conducted in moderate-to-severe TBI patients (Xiao et al., 2007; Wright et al., 2007; and Xiao et al., 2008), progesterone showed promising efficacy results in functional outcomes and mortality with an acceptable safety profile. BHR Pharma, LLC (supported by Besins Healthcare) is conducting the SyNAPSe ${ }^{\circledR}$ Phase 3 global trial to evaluate BHR-100, progesterone lipid infusion, in 1180 severe TBI patients (Glasgow Coma Scale 4-8).

Procedures: Subjects are entered into the SyNAPSe ${ }^{\circledR}$ study following proxy consent or per local country consent regulations, then randomized in a 1:1 ratio of BHR-100 or placebo. Randomization is conducted centrally, stratified by region. Subjects are treated with a loading dose of $0.71 \mathrm{mg} /$ $\mathrm{kg} / \mathrm{hr}$ of BHR-100 or placebo i.v. for the first hour followed by a continuous maintenance infusion of $0.5 \mathrm{mg} / \mathrm{kg} / \mathrm{hr}$ for a total of 120 hours $/ 5$ days. The infusion must be initiated within 8 hours of the brain injury, as confirmed by CT scan. The primary endpoint is the Glasgow Outcome Scale (GOS) assessed at 6-months post-injury. Secondary endpoints include the GOS-Extended, mortality, and the quality of life measure SF-36. The trial is registered at www. clinicaltrials.gov [ID NCT01143064].

Results: To date, the study has received approval in 15 countries, and 65 sites in the U.S., Latin America, Europe, and Asia have received Investigational Review Board/Ethics Committee approval to conduct the trial. Most of the additional 85 sites will be approved by May 2011. The study currently is under review in the People's Republic of China (PRC). An interim analysis is planned once 400 subjects (200 per treatment arm) complete the 6-month assessment.

Conclusion: BHR-100 is a promising candidate as a neuroprotectant for severe TBI patients. Successful completion of the SyNAPSe ${ }^{\circledR}$ Phase 3 study may lead to the approval and registration of BHR-100 as the first medication for the indication of TBI.

\section{Comparing the Predictive Value of Multiple Cognitive and Motor Tasks after Rodent TBI}

Zaorui Zhao, PhD, David J. Loane, Shruti V. Kabadi, Alan I. Faden, Department of Anesthesiology and the Center for Shock, Trauma and Anesthesiology Research (STAR), University of Maryland School of Medicine

Controlled cortical impact injury (CCI) is a widely used, clinically relevant model of traumatic brain injury (TBI). The mouse CCI model is particularly useful for studying the role of specific genes in the pathophysiology of TBI. Although functional outcomes have been used for years in this model, little work has been done to compare the predictive value of various cognitive and sensorimotor assessment tests, singly or in combination. Such informa- tion would be particularly useful for assessing mechanisms of injury or therapeutic intervention. Following isoflurane anesthesia, C57Bl/6 mice were subjected to sham, mild $(5.0 \mathrm{~m} / \mathrm{sec})$, moderate $(6.0 \mathrm{~m} / \mathrm{sec})$, or severe $(7.5 \mathrm{~m} / \mathrm{sec})$ CCI. A battery of behavioral tests were evaluated and compared. The beam walk task - performed at $0,1,3,7,14$, 21 , and 28 days post-injury (PID), showed good discrimination across severities of injury. The classical Morris water maze and reversal Morris water maze (rMWM) tests (PID14-23), as well as novel object recognition (PID24, 25), effectively discriminated spatial and novel object learning and memory across injury severities. Notably, the rMWM showed the greatest separation between mild and moderate/severe injury. The passive avoidance test (PID 27, 28) also well delineated differences in injury severity. This study shows the feasibility of using a larger number of complementary, functional outcome behavioral tests than traditionally employed to follow post-traumatic recovery for rodent TBI. We are currently comparing specific functional changes with cell loss across anatomical regions, as well as creating a new composite behavioral index that should be helpful in screening new neuroprotective agents or in investigating injury mechanism.

\section{Inhibition of Nitric Oxide Synthase Augmented Anti-depressant like Effects of Estradiol in Ovariectomized Mice: A New Strategy for Treatment of Depression}

Mehdi Ghasemi, MD, Department of Neurology, Johns Hopkins University; Pouria Heydarpour, Mohamad Salehi Sadaghiani, Mehrak Javadi Paydar, Mohsen Khosravi, Ahmad Reza Dehpour, Department of Pharmacology, School of Medicine, Tehran University of Medical Sciences, Tehran, Iran

Introduction: Ovarian hormones play a key role in mood regulation. Long-term ovarian hormone deprivation via ovariectomy (OVX) exerts depressive-like behavior in rodents. However, the exact mechanism underlying the antidepressant effects of ovarian hormones has not been yet completely understood. Nitric oxide (NO) signaling is also involved in the pathophysiology of depression. Several combinations of effective treatments have been used in the search for higher response rates than monotherapy to diminish depression in conditions of low ovarian hormones. The aim of this study was to investigate the antidepressant-like effects of nitric oxide signaling inhibition alone or combined with estradiol in OVX mice.

Methods: Female NMRI mice $(25-30$ g) were used throughout the study. After the onset of anesthesia, ovary 
and associated oviduct were removed in OVX group. In sham-operated animals, the parovarian fatty tissues and ovaries were just retracted and were replaced. Ten days later, mice were evaluated in the forced swimming test (FST). Animals were individually placed in an open cylindrical container of water. The duration of immobility was recorded during the last $4 \mathrm{~min}$ of the test $(6 \mathrm{~min})$. The analysis of variance (ANOVA) followed by Newman-Keuls post hoc test was used to analyze the data.

Results: Estradiol (0, 3, 10, $20 \mathrm{mg} / \mathrm{kg}$, s.c.), one hour after injection, exerted a dose-dependent anti-depressant like effects in OVX mice in the FST. The NO synthase inhibitor, L-NAME $(0,3,10,30 \mathrm{mg} / \mathrm{kg}$, i.v. $)$, also significantly reduced the immobility times of OVX mice in the FST. Administration of a sub-effective dose of L-NAME (10 mg/ $\mathrm{kg}) 15 \mathrm{~min}$ before a sub-effective dose of estradiol $(3 \mathrm{mg} /$ $\mathrm{kg}$ ) had a robust antidepressant-like effect in OVX mice. The NO precursor L-arginine $(750 \mathrm{mg} / \mathrm{kg}$, I.v., $30 \mathrm{~min}$ before estradiol treatment) prevented the antidepressant-like effect of potent doses of estradiol in OVX mice.

Conclusions: Inhibition of NO synthase as well as estradiol exerted a robust antidepressant-like effect in OVX mice. LArginine/NO pathway may be involved in the antidepressant-like effects of estradiol in OVX mice. Our data may provide a new insight into the treatment of depression in conditions of low ovarian hormone such as post-menopausal women.

The study was supported by a grant from Tehran University. There was no conflict of interest.

\section{Cost-effective Use of Biomarkers for Neurotherapeutic Agents: Quantitative Estimates from Historical Data}

\section{Orest Hurko, MD, The Biologics Group, Inc., Andrew Chadwick, Tessella PLC}

Biomarkers have been adopted widely in hopes of reducing development costs, but there has been no examination of which ones can do so. With the exception of blood pressure and lipids for prophylaxis of stroke, no other biomarkers have been recognized by regulators as surrogates that can substitute for clinical endpoints in pivotal trials of any neurotherapeutic agent. Thus, biomarker studies represent an additional cost which must be justified by quantification of the value of risk reduction, and/or implementation or sale of a companion diagnostic. We consider only the former in this study.

Our model for such quantification is based on published data from two sources: (a) the self reported mean phase length and out-of-pocket development costs of 68 new drugs randomly selected in 2001 by the Tufts Center for the Study of Drug Development from a survey of ten multinational pharmaceutical firms, and from (b) the mean success rates per clinical phase as reported by CMR International in 2005 . Net present value was calculated by discounting mean cash flow for each semester. Biomarkers were considered to belong into one of four classes: those predicting (1) efficacy or (2) toxicity, or those enabling selection of (3) responsive subjects or (4) effective dosing regimens.

Our sole metric was return on investment (ROI) realized by forgoing studies predicted by biomarkers to be futile. ROI for such go-no godecisions was a function of the cost of the biomarker program itself, the cost of capital, the net peak year earnings projected were the drug to be marketed, as well as the stage specific failure rate for a given therapeutic class. A key distinguishing feature was the predictive accuracy of each biomarker class. With few exceptions, the requirements for predictive accuracy of efficacy or toxicity biomarkers effectively preclude their economic use by a fully integrated large pharmaceutical company anticipating development of a blockbuster. In contrast, for a biotech any proof of effectiveness at an early stage may increase company valuation. Biomarkers for selection of doses or subjects may have a different impact on value because of a lower likelihood and impact of false alerts.

\section{Role of Cell Cycle in Experimental Spinal Cord Injury: Delayed Treatment with a Pan-CDK Inhibitor is Protective}

Junfang Wu, MD, PhD, Bogdan A. Stoica, Michael Dinizo, University of Maryland School of Medicine; Ahdeah Pajoohesh-Ganji, George Washington University Medical School; Alan I. Faden, MD, University of Maryland School of Medicine

Introduction: Traumatic spinal cord injury (SCI) causes tissue loss and associated neurological dysfunction through both mechanical damage and secondary biochemical and physiological responses. We have previously shown the role of cell cycle pathway in secondary injury following rat contusion SCI by examining effects a cell cycle inhibitor or gene knockout. In our prior study the non-selective CDK inhibitor flavopiridol was administrated centrally beginning 30 min after trauma. Such treatment significantly improved motor function and reduced lesion volume at 28 days after SCI.

Objective: In the present study, we investigate cell cycle pathway changes over time following SCI as well as the functional effects of cell cycle inhibition in rat contusion SCI model using delayed systemic administration of flavopiridol. Methods: Adult male rats were subjected to a moderate contusion SCI at T8. Flavopiridol was administrated by 
intraperitoneal injection beginning $24 \mathrm{~h}$ and continuing daily for 7 days. Expression of cell cycle proteins was assessed at various time point post-injury using immunoblot analysis. Behavioral assessments including the BBB scale and the combined behavioural score (CBS) were performed at days $1,7,14,21$, and 28 . At 28 days postinjury, lesion volume and spared white matter were assessed using histological analysis; stereological assessment of oligodendrocytes and neuron was also performed. Proliferation and activation of microglia and astrocytes were evaluated in both in vitro and in vivo.

Results: Immunoblot analysis shows a marked up-regulation of cell cycle-related proteins, including CDK4, E2F5, PCNA, cyclin $\mathrm{D} 1, \mathrm{pRb}$ at various time points after $\mathrm{SCI}$, whereas the endogenous CDK inhibitor p27 is down-regulated. Treatment with flavopiridol reduced cell cycle protein induction and increased p27 expression in injured spinal cord. Functional recovery was significantly improved from 7 days through 4 weeks after SCI. Treatment significantly reduced lesion volume and increased the area of spared white matter, as well as numbers of $\mathrm{CC} 1+$ oligodendrocytes. Furthermore, flavopiridol markedly inhibited proliferation of astrocytes and microglia, attenuating the production of factors associated with microglial activation and astrocytic reactivity in both in vivo and in vitro.

Conclusion: Our results support a role for cell cycle activation in the pathophysiology of SCI, and provide further support for the therapeutic potential of cell cycle inhibitors for the treatment of clinical SCI.

\section{Characterizing Radiology Reports in Patients with Alzheimer's Disease}

Prabhu Emmady, MD, David Gill, MD, Department of Neurology, Hershey Medical Center, Penn State University

Background: MTA is a radiologic finding seen in Alzheimer's dementia (AD) and mild cognitive Impairment and can help distinguish between other dementias. However the rate at which this finding on MRI's is assessed and reported by radiologists is unknown.

Design/Methods: Consecutive new referrals to the Penn State Hershey Memory and Cognitive Disorders Clinic that met the NINCDS-ARDA criteria for possible and probable AD between 09/2007 and 10/2010 were included in this retrospective study. Exclusion criteria included patients who could not get MRI. Each MRI was independently reviewed and interpreted by the behavioral neurologist. This was compared to the official MRI report, both body and impression.

Results: 111 patients were included in this retrospective study of which 50 had possible AD and 61 had probable
AD. Of these, 42 were males and 69 were females. The mean age of the patients was 75.6 years (38-91 years). 69 patients underwent an MRI at an academic medical center (AC) and 48 underwent MRI at a non-academic center (NAC). 6 had MRIs at both. MTA was mentioned in 14 $(20 \%)$ of the patients reported at the $\mathrm{AC}$ and $4(8 \%)$ of the patients reported at NAC. This difference however is not statistically significant. Only $2(1.8 \%)$ of all MRIs had grading of the MTA. Among the 111 patients, 32\% had grade 1 MTA, $29 \%$ had grade 2 MTA, $34 \%$ had grade 3 MTA and 5\% had grade 4 MTA. Brain atrophy however was reported in $84(75.6 \%)$ of all patients.

Conclusions: MTA is a finding that is relevant to the diagnosis of Alzheimer's disease and can be identified and graded, but rarely included in MRI reports. Hence, imaging must be reviewed by the clinician when caring for such patients and interpretation may be requested from radiologists with more experience in neuroimaging of dementia. Financial Sponsor: None. Dr. Emmady and Dr. Gill have no financial disclosures.

\section{Scientific and Economic Benefits of Sequential Parallel Comparative Design (SPCD), a Novel Clinical Trial Methodology}

Michael Knable, DO, Bethesda Behavioral Sciences; Matthias Bowman, RCTLogic

Introduction: For many illnesses, in order for a New Drug Application to be approved by the FDA, a study drug must be superior to placebo in at least 2 pivotal trials. Often, many trials are required before 2 positive trials are obtained. For example, the antidepressant paroxetine required 9 clinical trials to produce 2 positive results (1) and only 5 of 13 trials of fluoxetine submitted to the FDA were positive (2). It is estimated that the placebo response rate in trials for Major Depressive Disorder (MDD) is 35$45 \%$. A number of issues related to subject assessment and clinical trial conduct have been identified as possible contributors to high placebo response rates in trials for a wide range of illnesses, and a number of remedies have been proposed to minimize this effect (3). Sequential Parallel Comparative Design (SPCD) is a novel clinical trial method that has been employed in 4 completed trials to date (NCT 00683852, NCT 00555997, NCT 00321152, NCT 00955955) and will soon be employed in several additional trials, including a pivotal registration trial and a multi-site trial sponsored by NIH.

Methods: An SPCD trial involves two phases of treatment. Phase 1 is aimed at: (1) comparing drug (or any active treatment) and placebo, as in a conventionally designed single phase trial (with the expectation that the drug- 
placebo difference will be of "normal size") and (2) generating a cohort of placebo non-responders. Phase 2 is aimed at comparing drug and placebo, as in a conventionally designed single phase trial design, but utilizing individuals who were placebo non-responders in Phase 1 (with the expectation that the drug-placebo difference in Phase 2 will be the same or greater than in Phase 1). Sample sizes and power for a standard design and for SPCD were computed based on the asymptotic formulae for corresponding two-sided score tests with one degree of freedom, and with a two sided type I error rate of 0.05 . Data for the conventional design is based on having active treatment and placebo groups of equal size. Data for SPCD is derived from the Calculator available at RCTLogic.com, which was developed by Professor Anastasia Ivanova of the University of North Carolina. Such data is based on: (1) having, in phase 1, an active treatment group and two placebo groups, with patients allocated according to a 2:3:3 ratio; (2) a retention ratio (s) of 1 ; and (3) a ratio of treatment effects (r) of 1 (" $\mathrm{s}$ " and " $\mathrm{r}$ " are described on the Calculator page of RCTLogic.com, and in a manuscript by Professor Ivanova.) Further details of the statistical methods used in these estimations are available in previous publications (3-6)

Results: In trials using placebo, SPCD can permit a $20 \%$ $50 \%$ reduction of nwith no loss of "power", or a significant increase in "power". Calculated examples of sample size and statistical power will be presented in greater detail in the poster.

Conclusion: When compared to a standard design 300 patient trial, with an expected total cost of $\$ 30,000$ per patient, it is estimated that the SPCD design would result in direct cost savings of $\$ 1,800,000$, even if the reduction in nis only $25 \%$. It is also estimated that SPCD trial utilization would result in very large additional cost savings by allowing drugs to reach the market sooner and by avoiding the costs of "failed" trials. Software for estimation of SPCD effects is available at: www.RCTLogic.com.

\section{References:}

1. Hooper M, Amsterdam JD: Do clinical trials reflect drug potential? A review of FDA evaluation of new antidepressants. 39th Annual NCDEU Meeting, Boca Raton, June 11-14, 1998.

2. NDA 18-936, FDA 1988.

3. Fava M, et al.: The Problem of the Placebo Response in Clinical Trials for Psychiatric Disorders: Culprits, Possible Remedies, and a Novel Study Design Approach. Psychother Psychosom 72:115-127, 2003.

4. Grandi: The Sequential Parallel Comparison Model: A Revolution in the Design of Clinical Trials. Psychother Psychosom. 72:113-114, 20
Protective Effect of Beta-estradiol on Chemotherapeutic Agent Induced Demyelination in a New and Useful Vitro Model for Demyelination/Remyelination

Noelle Callizot, PhD, Maud Combes, Remy Steinschneider, Neuron Experts

Demyelination leads to a substantial functional impairment of the nervous system, reflecting the fact that the myelin sheath is an important phylogenetic invention to maintain the axon's functional and structural integrity. Major causes for demyelination are autoimmune inflammation, toxic agents (as antimitotic: vincristine, cisplatin, taxol\&), metabolic dysfunction, and mutations in genes related to the myelin sheath or other components of the nervous system.

Here, we used a reproducible in vitro demyelinationremyelination model based on primary cocultures of rat dorsal root ganglia (DRG) neurons and Schwann cells (SC). The cultures were maintained in neurobasal medium for 7 days in presence of NGF to allow SC to populate the DRG neurites. On day 7, cultures were supplemented with ascorbic acid to initiate basal lamina formation and myelination for 5 days. On day 12 of culture, test compounds (vincristine or cisplatin and Beta-estradiol) were incubated in standard neuronal medium with NGF and ascorbic acid for $24 \mathrm{~h}$ or $48 \mathrm{~h}$.

Vincristine and cisplatin (anti-mitotic agent) addition showed first a large demyelination followed by a neuronal death. States of demyelination and remyelination were observed by specific immunostaining of myelin components: myelin associated glycoprotein (MAG), myelin basic protein (MBP), peripheral myelin protein 22 (PMP22) and Caspr1 (contactin-associated protein 1) reflecting myelin internodes. Mature DRG neuron integrity was studied using neurofilament (NF) staining. Treatments of coculture model by Beta-estradiol were able to protect from demyelination induced by vincristine and cisplatin as well as from the cisplatin induced neurite death. These results suggest that Beta-estradiol has roles as neuroprotective agent against demyelination induced by chemotherapeutic agents. Betaestradiol could be a possible appropriate therapeutic strategy to interfere with the progress of the still incurable demyelinating disorders of both the peripheral and central nervous system.

\section{Single-chain Antibodies Targeted Against Alpha-Synuclein Protect Against Dopamine Neuron Cell Death}

Kathleen Maguire-Zeiss, $\mathrm{PhD}$, Georgetown University Medical Center; William Bowers, University of Rochester School of Medicine and Dentistry; Howard J. Federoff, $\mathrm{MD}, \mathrm{PhD}$, Georgetown University Medical Center 
Alpha-synuclein has been linked to both familial and sporadic Parkinson's disease (PD) as well as other synucleinopathies. Point mutations in and multiplications of the $\alpha$-synuclein gene, SNCA, are associated with familial forms of PD while recent GWAS demonstrate a risk associated with SNCA polymorphisms in sporadic disease. Human genetic data, tissue culture and animal studies all point to a toxic gain of function for $\alpha$ synuclein in disease pathogenesis. More specifically, this $14 \mathrm{kDa}$ protein can exist in a random coil conformation but has a proclivity to misfold into toxic oligomers. Since toxicity is intimately linked to the conformation of this protein we sought to target the toxic moieties as a novel therapeutic approach to decrease $\alpha$-synucleinmediated cell death.

First, we identified human single chain antibodies that recognize different conformations of $\alpha$-synuclein including one that binds dopamine-modified protein. This is relevant since dopamine modified $\alpha$-synuclein forms stable presumably toxic oligomers. Second, we engineered recombinant adeno-associated vectors to overexpress the anti-synuclein single chain antibodies for gene therapy ( $\mathrm{rAAVscFv}$ ). We next stereotaxically injected the substantia nigra of transgenic mice that overexpress human $\alpha$-synuclein with the rAAVscFvs. Seven months post-injection mouse brains were harvested and subjected to immunohistochemistry for tyrosine hydroxylase and substantia nigra dopamine neurons were enumerated using unbiased stereology. Here we report the identification of $\alpha$-synuclein-specific single chain antibodies which following stereotaxtic rAAV delivery result in a significant increase in tyrosine hydroxylase-positive dopamine neurons compared to control injected $\alpha$-synuclein transgenic mice.

\section{Improving Signal Detection in CNS Clinical Trials: Lessons Learnt and a Methodology to Assess Site Performance}

\section{Amir Kalali, MD, Sonia Davis, Quintiles}

Signal detection in CNS trials has come under intense scrutiny recently, due to the number of failed trials. Methodological issues impacting signal detection are particularly important in CNS clinical trials due to the nature of the disorders being studied and the outcome measures used. This area is particularly hard to study as there is little good quality data to illuminate the issues. This presentation discusses factors that have been found to impact signal detection, as well as a statistical methodology to assess clinical trial sites in terms of signal detection.

\section{Opportunities for Evaluating Novel Memory Enhancers in Man}

Keith A. Wesnes, $\mathrm{PhD}$, Chris J. Edgar, $\mathrm{PhD}$, United BioSource Corporation, Goring, UK

While the field of evaluating cognition enhancers in man has been served well by measuring memory retention over relatively brief (10 to 30 minute) intervals and also using the scopolamine model, other paradigms are required to evaluate compounds which can affect processes such as long-term potentiation or which promote neurogenisis. This paper will describe some experimental procedures which can be used to evaluate such compounds in man.

A number of novel compounds including various phosphodiesterase inhibitors may improve memory via mechanisms which can be temporally remote from the initial learning experience. For such compounds, the typical paradigm in human cognitive psychopharmacology of testing retention soon after initial registration of the information is not helpful, and recall and recognition need to be tested over much longer periods (eg 24 to 72 hours). Such testing paradigms will be described, and work on massed versus spaced learning to support the concept that longer term episodic memory can be enhanced independently from shorter term episodic memory. Furthermore, data will be presented from Alzheimers patients showing the capability to perform recognition memory tests for verbal and pictorial information 24 hours after initial presentation and registration.

Other compounds may enhance memory by promoting neurogenesis in the dentate gyrus. Visual pattern recognition paradigms will be described which have been shown by neuroimaging techniques to involve dentate gyrus activity. Data showing selective age-related declines in aspects of picture recognition task performance compared to verbal recognition will be presented, suggesting a paradigm for evaluating compounds which target neurogenesis.

Further, a wide variety of aspects of memory and attention show marked declines decade by decade from the $20 \mathrm{~s}$ onwards, even in healthy individuals, providing easily accessible volunteer pools for a variety of potential cognition enhancers. Automated testing permits the speed of retrieval to be assessed in working memory and episodic recognition tasks, aspects of function which decline rapidly in normal and pathological aging, but which are generally overlooked by traditional neuropsychological tests. For example aspects of spatial working memory decline by two or more standard deviations by middle age, which could provide an opportunity to evaluate compounds found in pre-clinical studies to act upon this type of memory.

In conclusion, experimental paradigms involving cognitive testing can be tailored to assess compounds which may 
enhance memory via a variety of mechanisms. Such paradigms can be used in Phase I and Phase IIa studies to provide early evidence of proof of principle, and in later stage trials as efficacy measures.

\section{Antidepressant Treatment Does Not Affect the Anti-Chorea Efficacy of Tetrabenazine in TetraHD, a Double-Blind Placebo-Controlled Trial}

Fred Marshall, MD, Shirley Eberly, MA, University of Rochester; Nicolas Scoglio, BS, Ira Shoulson, MD, University of Rochester Medical Center; Kathleen Clarence-Smith, MD, $\mathrm{PhD}$, KM Pharmaceutical Consulting

Depression is common in Huntingtons disease (HD), and diagnoses ranging from dysthymia to major depression have been reported in $11 \%$ to $55 \%$ of HD patients (De Marchi and Menella, 2000). We sought to determine whether treatment with an antidepressant (ATD) could affect the antichorea efficacy and optimal dosage of tetrabenazine (TBZ) by conducting a post-hoc analysis of TetraHD, a 12-week, double-blind, parallel-group, placebocontrolled clinical trial (Marshall et al., 2006). Optimal dosing ranged from $12.5 \mathrm{mg}$ /day to $100 \mathrm{mg} /$ day, and was determined by a slow upward adjustment followed as needed by downward adjustment. A total of 84 subjects were enrolled, 54 were randomized to TBZ and 30 were randomized to placebo (PLA). A responder was prespecified as a participant with a e3 reduction in Total Chorea Score. Only patients with a Week 12 visit were included in this post-hoc analysis (TBZ:51; PLA:29). At baseline, 28 of $51(55 \%)$ subjects randomized to TBZ, and 19 of $29(65 \%)$ subjects randomized to placebo were receiving an ATD. In the TBZ group, dosage was not affected by baseline ATD use (no ATD: 37.5 to $100 \mathrm{mg}$ / day; on ATD: 25 to $100 \mathrm{mg}$ /day; Cochran-Armitage trend test, $\mathrm{p}=0.5512$ ). In a multivariate model including all 80 subjects, baseline ATD use was not a significant predictor of change in Total Chorea Score $(p=0.1137)$. In the TBZ group, the magnitude of antichorea efficacy was not significantly affected by concomitant ATD (no ATD:-4.61 \pm 0.91; on ATD:-6.21 \pm 0.80$)$. A similar finding was observed in the PLA group (no ATD: $-0.13 \pm 1.45$; on ATD: $-2.06 \pm 0.98$ ). In the TBZ group, baseline ATD did not significantly affect response rate (no ATD: 61\%; on ATD: 75\%). Among the 35 TBZ responders, dose was not significantly different for those on or not on baseline ATD (Cochran-Armitage trend test, $\mathrm{p}=0.8570$ ). A similar finding was observed in the $16 \mathrm{TBZ}$ non-responders (Cochran-Armitage trend test, $\mathrm{p}=0.5860$ ). In conclusion, baseline use of ATDs was found not to affect TBZ dosage or antichorea efficacy in this doubleblind, placebo-controlled study.
De Marchi N, Menella R. Huntington's disease and its association with psychopathology. Harvard Rev Psychiatry 2000; 7:278-89.

Marshall F and the Huntington Study Group. Tetrabenazine as antichorea therapy in Huntington disease: A randomized controlled trial. Neurology. 2006;66:36672.

Disclosures: Dr. Marshall received travel reimbursement from Prestwick Pharmaceuticals to present data at the FDA advisory committee meeting on TBZ prior to its approval for marketing in the US. Dr. Shoulson received consulting honoraria from Prestwick Pharmaceuticals and Lundbeck Inc after publication of the cited primary report on TBZ (Marshall et al., 2006). Dr. Clarence-Smith was employed by Prestwick Pharmaceuticals and is a paid consultant to Lundbeck Inc., which markets TBZ in the United States as Xenazine.

\section{2-deoxy-D-glucose reduces progression of volumetric and diffusion tensor MRI abnormalities in a rat model of TBI}

Thomas Sutula, MD, PhD, Elizabeth Hutchinson, $\mathrm{PhD}$, Department of Neurology, University of Wisconsin; Elizabeth Meyerand, PhD, Department of Medical Physics, University of Wisconsin; Paul Rutecki, MD, Department of Neurology, University of Wisconsin

Inhibition of glycolysis may have widespread neuroprotective actions in the brain as it has been shown to be both an anti-convulsant and anti-epileptic agent as well as protective following ischemia. This profile of broad efficacy suggests that the glycolytic inhibitor 2-deoxy-D-glucose (2DG) is well suited to treat the collection of pathological changes that evolve following traumatic brain injury (TBI). In order to investigate the effects of $2 \mathrm{DG}$ in a controlled cortical impact (CCI) rat model of TBI, serial in-vivo magnetic resonance imaging (MRI) and diffusion tensor MRI (DTI) scans were collected at 1 week, 1 month and 6 months following CCI in 2DG treated animals and saline treated controls. Brief treatment with $2 \mathrm{DG}$ at $40 \mathrm{mg} / \mathrm{kg}$ IP $30 \mathrm{~min}$ prior to $\mathrm{CCI}$, and then $250 \mathrm{mg} / \mathrm{kg}$ immediately after and twice daily for 2 weeks reduced the progression of structural abnormalities measured by multiple imaging markers of damage including progressively increasing ventricle volume, increased hippocampal mean diffusion (MD) and evolving patterns of hippocampal and corpus callosum fractional anisotropy (FA). The proportional change in ventricle volume from 1 week to 1 month following injury was reduced by $70 \%$ in the group treated with $2 \mathrm{DG}$ compared to the group treated with saline ( $\mathrm{p}=$ 0.015 ) and there were greater ipsilateral increases in 
hippocampal MD from 1 week to 6 months following CCI for saline $(p=0.0026)$ than $2 \mathrm{DG}$ treated $(p=0.289)$ groups. Additionally, a temporal profile of increased FA in the anterior corpus callosum from 1 week to 6 months following CCI was found for sham injured animals $(p=$ $0.0274)$ and animals treated with $2 \mathrm{DG}(\mathrm{p}=0.0099)$, but not for saline treated animals $(p=0.8396)$. The MRI and DTI measures used in this study appear to be useful biomarkers for TBI progression and for the assessment of therapies and these markers revealed that focal and generalized structural alterations progressively evolve for as long as 6 months after TBI and are reduced by glycolytic inhibition.

This work was funded by the Department of Defense Hypothesis Development Award DR080424.

\section{The Range Disparity Distribution: A New Statistical Tool with Practical Applications in Clinical Trials}

Andrew R. Blight, PhD, ${ }^{1}$ Lawrence Marinucci, MS, ${ }^{1}$ Thomas Wessel, MD, ${ }^{1}$ Joel Waksman, $\mathrm{PhD},{ }^{2}$ Tai Xie, $\mathrm{PhD},{ }^{2}$ Ron Cohen, $\mathrm{MD}^{1}$

${ }^{1}$ Acorda Therapeutics Inc.,

${ }^{2}$ Brightech International LLC,

Background: Conventional approaches to responder analysis generally rely on an arbitrary fixed threshold of change to define a "meaningful" improvement. Such thresholds are vulnerable to background variability and usually must be selected using existing data to define expected spontaneous variability, in order to limit false positives. We have developed and describe the properties of a novel statistical tool, the Range Disparity Distribution (RDD), and a response definition that together allow a priori determination of the frequency of expected false positive responses due to random variation (i.e. not true "placebo responses").

Methods: We developed proofs that the RDD describes the probability of a given number of items in one set of sample measurements $(\mathrm{T})$ falling outside the range of all the items in another set of sample measurements (S), regardless of the underlying population distribution, provided that the measurements are from a continuous random variable. For the RDD (Y), the mean can be described by the equation $\mathrm{E}$ $(\mathrm{Y})=\mathrm{T} /(\mathrm{S}+1)$, and the variance is represented as $\operatorname{var}(\mathrm{Y})=$ $\mathrm{ST}(\mathrm{S}+\mathrm{T}+1) /(\mathrm{S}+1)^{2}(\mathrm{~S}+2)$, with the derivation of this distribution identical to the negative binomial distribution, except that sampling is without replacement.

Results: Using the RDD, it is possible to pre-specify a series of measurements and a criterion for treatment response (based on consistently exceeding the range of off-treatment values) that sets, a priori, the desired frequency of false positives expected from random variation. Clinical trial data, obtained using these methods, demonstrated that non-responders on active treatment showed a distribution of change almost identical to the distribution for the placebo-treated group, indicating a true separation of treatment-related response. In contrast, a conventional responder analysis, using an arbitrary threshold of average change, resulted in non-responders with a distribution of change clearly different from placebo. The RDD can also be used to provide independent statistical testing of upper and lower limits of sample distributions, even with small sample sizes.

Conclusions: The RDD provides a new statistical tool that can be of particular benefit for prospectively defining response criteria with several advantages over conventional criteria. It may be useful in a wide variety of applications in the neurosciences.

Study supported by Acorda Therapeutics, Inc.

\section{Immunoregulation in Parkinson's Disease}

Jessica A.L. Hutter Saunders ${ }^{1}$, Lisa M. Kosloski ${ }^{1}$, Pam Santamaria $^{2}$, Scott Goodman ${ }^{2}$, Diego R. Torres-Russotto ${ }^{3}$, David G. Standaert ${ }^{4}$, R. Lee Mosley ${ }^{1}$, Howard E. Gendelman ${ }^{1}$ ${ }^{1}$ Department of Pharmacology and Experimental Neuroscience, University of Nebraska Medical Center;

${ }^{2}$ Neurological Consultants of Nebraska;

${ }^{3}$ Department of Neurological Sciences, University of Nebraska Medical Center;

${ }^{4}$ Department of Neurology, University of Alabama at Birmingham

Neuroinflammation is an integral characteristic of nigrostriatal degeneration in Parkinson's disease (PD), yet current therapies are palliative and do not halt or slow neuronal loss. Both innate and adaptive immune responses increase the extent and progression of neurodegeneration in 1methyl-4-phenyl-1,2,3,6-tetrahydropyridine (MPTP) intoxicated mice. Aggregated and aberrant species of a-synuclein (a-syn), including nitrated a-syn ( $\mathrm{N}-\mathrm{a}-\mathrm{syn})$ are found in the extra-neuronal milieu of the CNS and induce a neurotoxic inflammatory microglial response. Furthermore, N-a-syn, but not unmodified a-syn, activates antigen-presenting cells and induces robust effector $\mathrm{T}$ cell (Teff, i.e. Th1 and Th17) responses that exacerbate microglial activation and accelerate nigrostriatal dopaminergic neuron loss. Moreover, regulatory $\mathrm{T}$ cells (Treg) ameliorate both innate and Teffmediated inflammation and subsequent neuronal degeneration. Based on these observations in vitro and in animal models of $\mathrm{PD}$, we hypothesized perturbations in peripheral immune phenotype and/or function of PD patients. For 
these studies, whole blood samples were collected from PD patients between 3 and 7 years of diagnosis and their caregivers as age- and environmental-matched controls. Flow cytometric analysis of peripheral blood mononuclear cells demonstrated alterations in Treg and Teff phenotypes and frequencies in PD patients compared to caregiver controls. While CD45RO expression was increased, CD45RA expression was concomitantly reduced on CD4+ CD25+CD127- Treg from PD patients suggesting an increase in memory Treg and a decrease in naïve Treg. Expression of the E-cadherin-specific receptor, CD103, was significantly reduced on CD4+CD25+CD127+ Teff from PD patients compared to those from caregivers. Furthermore, the overall frequency of Teff in PD patients was significantly increased when compared to caregivers, while no significant differences in Treg frequencies were detected, suggesting possible dysregulation of Teff in PD patients. To assess their regulatory potential, Treg were isolated by fluorescence-activated cell sorting from the whole blood of PD patients and caregivers. In vitro CFSE-based proliferation assays show differences in the suppressive function of Treg amongst paired subjects. These data are supportive of our hypothesis that $\mathrm{T}$ cellmediated immunity plays a significant role in PD pathogenesis and/or progression, and provides candidate targets for immunomodulatory therapies to mitigate disease progression in PD.

\section{Sham Control Groups for Evaluation of Neurosurgery Delivered Therapeutics: The Patient View}

\section{Perry D. Cohen, PhD, Parkinson Pipeline Project}

Double blind placebo controlled trials are considered to be the gold standard for rigorous assessment of the efficacy of a new therapy for FDA approval, but the FDA law requires randomized, well controlled trials, not blinded, placebo controls. Our previous analysis of failures of clinical trials showed a similar pattern of results between the open label safety study and the sham controlled pivotal trial in four consecutive therapies. That paper was in part responsible for the instigation of an international conference sponsored by the NIH on the ethical and practical issues in the controversial practice of using sham brain surgery control groups in the design of clinical trials. This conference was unique in the invitation of a patient perspective along with the expert views of medical ethicists, statisticians, neurologists and neurosurgeons. The patient conclusions were quite different than the predominant views of clinical scientists who argue that sham arms were mandatory to control for the very large placebo response that is produced by brain surgery. Patients view placebo response as potential beneficial therapy, which may interact with the treatment.

This presentation identifies the differences between patients' perspectives and scientists' perspectives in three areas and analyzes them to account for the differences in conclusions.

1) Time - delay has negative consequences for patients whose alternative is continued progression and suffering.

2) Error tolerance - scientists limit false positives versus patients prefer to limit false negatives.

3) Bias - scientists consider psychological placebo response from expectations as bias to be eliminated versus patients who view expectations and hope as part of positive healing processes.

We argue that in an environment that is increasingly oriented to patient centered approaches in order to successfully address fundamental psychological components of chronic disease that the patient perspective has been missing and should be included.

\section{Drug Discovery Program in Dystonia}

Ellen J. Hess, Cicely Toomer, H.A. Jinnah, Departments of Pharmacology and Neurology, Emory University School of Medicine

Dystonia is characterized by excessive involuntary muscle contractions leading to twisting movements and unnatural postures. It has many different clinical manifestations, and many different causes. More than 3 million people worldwide suffer from dystonia, yet there are few broadly effective treatments. The relative scarcity of effective small molecule drugs together with the prevalence of primary and secondary dystonias provides a tremendous opportunity for the development and introduction of novel therapeutics. Progress in research has advanced our understanding of dystonia to a point where drug discovery efforts are now feasible, particularly because there has been an explosion in the development of rodent model of dystonia. We have created a drug-screening program using three different mouse models of dystonia. The models used in our screen arise from different etiologies and underlying mechanisms. Therefore, compounds effective in several models may prove to be broadly effective across various forms of dystonia. Models included in the screen are 1) dystonia induced by the calcium channel agonist Bay K 8644; 2) the tottering mouse mutant which exhibits attacks of dystonia caused by a mutation in the P/Q-type calcium channel and; 3) a knockin mouse that carries the mutation that causes early onset torsion dystonia (DYT1). Compounds are tested in vivo in all three different mouse models of dystonia. A 
rating scale similar to those used to assess dystonia in humans is used to determine responses in the Bay K 8644 and tottering mouse models, the two models that exhibit dystonia. This scale was optimized for inter-rater reliability $(0.9+)$, effect size $(\geq 3)$ and throughput, so even drugs with modest efficacy can be identified quickly. An assessment of walking patterns using the Digigait analysis system is used to determine efficacy in the Dyt1 knockin mouse model, because this model does not exhibit overt dystonia. We are currently testing FDA-approved drugs, so candidates can reach patients quickly. Additionally, compounds of interest that arise in academic or pharmaceutical settings can be quickly incorporated into this screen.

Funded by The Bachmann-Strauss Dystonia \& Parkinson Foundation. The authors have no personal financial interests. 